\title{
An obstruction to the strong relative hyperbolicity of a group
}

\author{
James W. Anderson, Javier Aramayona and Kenneth J. Shackleton*
}

25 December, 2006

\begin{abstract}
We give a simple combinatorial criterion for a group that, when satisfied, implies the group cannot be strongly relatively hyperbolic. Our criterion applies to several classes of groups, such as surface mapping class groups, Torelli groups, and automorphism and outer automorphism groups of free groups.
\end{abstract}

MSC 20F67 (primary), 20F65 (secondary)

\section{Introduction}

In recent years, the notion of relative hyperbolicity has become a powerful method for establishing analytic and geometric properties of groups. Relatively hyperbolic groups, first introduced by Gromov [11] and then elaborated on by various authors (see Farb [7], Szczepański [21], Bowditch [3]), provide a natural generalization of hyperbolic groups and geometrically finite Kleinian groups.

When a finitely generated group $G$ is strongly hyperbolic relative to a finite collection $L_{1}, L_{2}, \ldots, L_{p}$ of proper subgroups, it is often possible to deduce that $G$ has a given property provided the subgroups $L_{j}$ have the same property. Examples of such properties include finite asymptotic dimension (see Osin [18]), exactness (see Ozawa [20]), and uniform embeddability in Hilbert space (see Dadarlat and Guentner [5]). In light of this, identifying a strong relatively hyperbolic group structure for a given group $G$, or indeed deciding whether or not one can exist, becomes an important objective.

The main result of this note, Theorem 2 in Section 3, asserts that such a structure cannot exist whenever the group $G$ satisfies a simple combinatorial property, namely that its commutativity graph with respect to some generating set $S$ is connected. We describe this graph in Section 3.

We then give elementary proofs that many groups of interest do not admit a strong relatively hyperbolic group structure. This is the case for all but finitely many surface mapping class groups, the Torelli group of a closed surface of genus at least 3, and the (special) automorphism and outer automorphism groups of almost all free groups. We note that this has already been established for many of these groups by other methods, and we indicate this where appropriate.

\footnotetext{
* Supported by an EPSRC studentship
} 
Acknowledgements. Parts of this paper were written during visits of the second author to the Bernoulli Center, Lausanne, and the third author to the Tokyo Institute of Technology. Both authors wish to express their gratitude to these institutions. We would like to thank Martin Bridson for showing us a way of extending the initial results of Section 4.3 to the cases $n \in\{3,4\}$. We would also like to thank Martin Dunwoody and Ian Leary for helpful conversations, and Igor Belegradek and Mark Sapir for comments on a previous version of this paper. Finally, we would like to thank the referee for his or her comments and suggestions.

\section{Relatively hyperbolic groups}

All groups appearing in this note are infinite, unless otherwise stated.

There are two related but inequivalent definitions of relative hyperbolicity, after Gromov [11], that are commonly used, one developed by Farb [7], and the other developed by Szczepański [21] and by Bowditch [3]. As we only go into as much detail as is required for us to state our results, we refer the interested reader to the cited papers for an extensive treatment.

We first give the definition given by Farb [7] and refer to this as weak relative hyperbolicity. For a group $G$, a finite generating set $S$ and a finite family of proper finitely generated subgroups $\left\{L_{1}, L_{2}, \ldots, L_{m}\right\}$, we form an augmentation $\operatorname{Cay}^{*}(G, S)$ of the Cayley graph $\operatorname{Cay}(G, S)$ as follows: Give $\operatorname{Cay}(G, S)$ the path-metric obtained by declaring each edge to have length 1 . Then, for each $1 \leq j \leq m$, adjoin to $\operatorname{Cay}(G, S)$ a new vertex $v_{g L_{j}}$ for each coset $g L_{j}$ of $L_{j}$ and declare the distance between each new vertex $v_{g L_{j}}$ and each vertex in the associated coset $g L_{j}$ to be 1 . We say that $G$ is weakly hyperbolic relative to $L_{1}, L_{2}, \ldots, L_{m}$ if the resulting metric on $\operatorname{Cay}^{*}(G, S)$ is hyperbolic in the sense of Gromov. This definition does not depend on the choice of generating set.

In the same paper, Farb introduces the bounded coset penetration property (BCP), satisfied by many important examples of weakly relatively hyperbolic groups. Roughly speaking, BCP imposes certain fellow-travelling conditions on pairs of quasi-geodesics on $\operatorname{Cay}(G, S)$ with the same endpoints that enter cosets of the subgroups $L_{j}, 1 \leq j \leq m$.

Bowditch [3] gives two equivalent dynamical notions of relative hyperbolicity, of which we recall the second. We will refer to this notion as strong relative hyperbolicity. We say that a group $G$ is strongly hyperbolic relative to the family $L_{1}, L_{2}, \ldots, L_{m}$ of proper finitely generated subgroups if $G$ admits an action on a connected, hyperbolic graph $\mathcal{G}$ such that $\mathcal{G}$ is fine (that is, for each $n \in \mathbb{N}$, each edge of $\mathcal{G}$ belongs to only finitely many circuits of length $n$ ), there are only finitely many $G$-orbits of edges, each edge stabiliser is finite, and the stabilisers of vertices of infinite valence are precisely the conjugates of the $L_{j}$.

We note that strong relative hyperbolicity, with respect to some finite collection of proper finitely generated subgroups, is equivalent to weak relative hyperbolicity plus BCP, with respect to the same collection of subgroups (see Dahmani [6]). As noted by Szczepański [21], the group $\mathbb{Z} \oplus \mathbb{Z}$ is weakly hyperbolic, though not strongly hyperbolic, relative to the diagonal subgroup $\{(m, m): m \in \mathbb{Z}\}$. 


\section{The commutativity graph}

We begin by describing a graph which attempts to capture the notion that a group is well generated by large abelian subgroups. This graph, and generalizations, appears in Karlsson and Noskov [15] and in Farb and Franks [8]. A more general graph, expressed in terms of minipotent words, appears in Culler and Vogtmann [4].

Definition 1 (Commutativity graph) Let $G$ be a group and let $S$ be a (possibly infinite) generating set for $G$, all of whose elements have infinite order. The commutativity graph $K(G, S)$ for $G$ with respect to $S$ is the simplicial graph whose vertex set is $S$ and where any two distinct vertices $s$, $s^{\prime}$ are connected by an edge if and only if there are non-zero integers $n_{s}, n_{s^{\prime}}$ such that $\left\langle s^{n_{s}},\left(s^{\prime}\right)^{n_{s^{\prime}}}\right\rangle$ is abelian.

So long as there is no risk of confusion, we will use the same notation for elements of $S$ and vertices of $K(G, S)$. Typically we shall only consider commutativity graphs in which adjacent vertices, rather than powers of adjacent vertices, commute.

Our main result, giving an obstruction to the strong relative hyperbolicity of a group, may be stated as follows.

Theorem 2 Let $G$ be a finitely generated group. Suppose there exists a (possibly infinite) generating set $S$ of cardinality at least 2 such that every element of $S$ has infinite order and $K(G, S)$ is connected. Suppose further that there exist adjacent vertices $s, s^{\prime}$ of $K(G, S)$ and non-zero integers $n_{s}, n_{s^{\prime}}$ such that $\left\langle s^{n_{s}},\left(s^{\prime}\right)^{n_{s^{\prime}}}\right\rangle$ is a rank 2 free abelian group. Then, $G$ is not strongly hyperbolic relative to any finite collection of proper finitely generated subgroups.

The remainder of this section is dedicated to proving Theorem 2. The main tool we use is the following theorem on virtual malnormality for strongly relatively hyperbolic groups, which is contained in the work of Farb [7] and Bowditch [3], and is explicitly stated as Theorem 1.4 and Theorem 1.5 of Osin [19].

Theorem 3 Let $G$ be a finitely generated group that is strongly hyperbolic relative to the proper finitely generated subgroups $L_{1}, \ldots, L_{p}$. Then,

1. For any $g_{1}, g_{2} \in G$, the intersection $g_{1} L_{j} g_{1}^{-1} \cap g_{2} L_{k} g_{2}^{-1}$ is finite for $1 \leq j \neq k \leq p$.

2. For $1 \leq j \leq m$, the intersection $L_{j} \cap g L_{j} g^{-1}$ is finite for any $g \notin L_{j}$.

Remark 4 Theorem 3 immediately implies that if $g \in G$ has infinite order and if $g^{k}$ lies in a conjugate $h L_{j} h^{-1}$ of some $L_{j}$, then $g$ lies in the same conjugate $h L_{j} h^{-1}$ of $L_{j}$.

We also need the following lemma, which follows directly from Theorem 4.16 and Theorem 4.19 of Osin [19]. 
Lemma 5 Let $G$ be a finitely generated group that is strongly hyperbolic relative to the proper finitely generated subgroups $L_{1}, L_{2}, \ldots, L_{p}$. If $A$ is a free abelian subgroup of $G$ of rank at least 2 , then $A$ is contained in a conjugate of one of the $L_{j}$.

We are now ready to prove Theorem 2 .

Proof [of Theorem 2] Suppose for contradiction that $G$ is strongly hyperbolic relative to the finite collection $L_{1}, L_{2}, \ldots, L_{p}$ of proper finitely generated subgroups. We first show that no conjugate of any $L_{j}$ can contain a non-zero power of an element of $S$. So, suppose there is some $g \in G$, some $s_{0} \in S$, and some integer $k \neq 0$ such that $s_{0}^{k} \in g L_{j} g^{-1}$ for some $1 \leq j \leq p$. (Note that, by Remark 4, this implies that $s_{0} \in g L_{j} g^{-1}$ as well.)

Let $s_{1}$ be any vertex of $K(G, S)$ adjacent to $s_{0}$. As there are non-zero integers $n_{0}, n_{1}$ such that $\left\langle s_{0}^{n_{0}}, s_{1}^{n_{1}}\right\rangle$ is abelian, we see that $\left\langle s_{0}^{n_{0}}\right\rangle \subseteq g L_{j} g^{-1} \cap s_{1}^{n_{1}} g L_{j} g^{-1} s_{1}^{-n_{1}}$. However, since the subgroup $\left\langle s_{0}^{n_{0}}\right\rangle$ of $G$ is infinite, Theorem 3 implies that $s_{1}^{n_{1}} \in g L_{j} g^{-1}$. By Remark 4, we see that $s_{1} \in g L_{j} g^{-1}$.

Let $s$ be any element of $S$. By the connectivity of $K(G, S)$, there exists a sequence of elements $s_{0}, s_{1}, \ldots, s_{n}=s$ of $S$ such that $s_{k-1}$ and $s_{k}$ are adjacent in $K(G, S)$ for each $1 \leq k \leq n$. The argument given above implies that if $s_{k-1} \in g L_{j} g^{-1}$, then $s_{k} \in g L_{j} g^{-1}$. In particular, we have that $s \in g L_{j} g^{-1}$. Since $G$ is generated by $S$, it follows that $G$ and $g L_{j} g^{-1}$ are equal, contradicting the assumption that the subgroup $L_{j}$ is proper. We conclude that if $G$ is strongly hyperbolic relative to $L_{1}, L_{2}, \ldots, L_{p}$ then no conjugate of any $L_{j}$ can contain a non-zero power of an element of $S$.

By assumption, there exist adjacent vertices $t$ and $t^{\prime}$ of $K(G, S)$ for which there exist non-zero integers $n_{t}, n_{t^{\prime}}$ such that $A=\left\langle t^{n_{t}},\left(t^{\prime}\right)^{n_{t^{\prime}}}\right\rangle$ is a rank 2 free abelian group. According to Lemma 5, we see that $A$ must lie in some conjugate of some $L_{j}$. In particular, this conjugate of $L_{j}$ contains a non-zero power of an element of $S$, and we have a final contradiction.

QED

\section{Applications}

In this section, we apply Theorem 2 to a selection of finitely generated groups, and deduce that each is not strongly hyperbolic relative to any finite collection of proper finitely generated subgroups. We shall say that such a group is not strongly relatively hyperbolic.

\subsection{Mapping class groups}

Let $\Sigma$ be a connected and oriented surface without boundary, of finite topological type and negative Euler characteristic. The mapping class group $\operatorname{MCG}(\Sigma)$ associated to $\Sigma$ is the group of all homotopy classes of orientation preserving self-homeomorphisms of $\Sigma$. (For a thorough account of these groups, we refer the reader to Ivanov [13].) It is known that every mapping class group MCG $(\Sigma)$ is finitely presentable and can be generated by Dehn twists. Masur and Minsky [16] prove that $\operatorname{MCG}(\Sigma)$ is weakly hyperbolic relative to a finite collection of curve stabilisers.

Let $S$ be the collection of all primitive positive Dehn twists about simple closed curves on $\Sigma$. Note, an element of a group is said to be primitive if it is not the second or higher power of any element 
of the group. The associated commutativity graph is precisely the 1-skeleton of the curve complex (see Harvey [12] for a definition), following from the observation that two non-trivial Dehn twists commute if and only if their associated curves are disjoint. The curve complex is connected provided $\Sigma$ is not a pair of pants, a once-punctured torus or a four-times punctured sphere. Moreover, the Dehn twists associated to any pair of adjacent vertices in the curve complex generate a rank 2 free abelian group. We thus have the following:

Proposition 6 Let $\Sigma$ be a connected and oriented surface without boundary, of finite topological type and negative Euler characteristic. If $\Sigma$ is not pair of pants, a once-punctured torus or a four-times punctured sphere, then the mapping class group $\operatorname{MCG}(\Sigma)$ of $\Sigma$ is not strongly relatively hyperbolic.

We note that the mapping class group of a pair of pants is trivial and that, when $\Sigma$ is a oncepunctured torus or a four-times punctured sphere, $\operatorname{MCG}(\Sigma)$ is isomorphic to $\operatorname{PSL}(2, \mathbb{Z})$ which is a hyperbolic group.

The statement of Proposition 6 is implicit in both Karlsson and Noskov [15] and Bowditch [3], using convergence groups. Another proof, by Behrstock, Druţu, and Mosher [1], uses asymptotic cones and the description of relative hyperbolicity due to Druţu and Sapir.

Since the mapping class group of a punctured sphere can be viewed as a braid group, the braid group $B_{n}$ on $n$ strings is not strongly relatively hyperbolic whenever $n \geq 5$. This also follows by considering the usual presentation for $B_{n}$ and its corresponding commutativity graph.

\subsection{Torelli groups}

The Torelli group $\mathcal{I}(\Sigma)$ of a connected and oriented surface $\Sigma$ is the kernel of the natural action of the mapping class group $\operatorname{MCG}(\Sigma)$ on the first homology group $H_{1}(\Sigma, \mathbb{Z})$. It is of infinite index in $\operatorname{MCG}(\Sigma)$. If $\Sigma$ is closed and has genus at least $3, \mathcal{I}(\Sigma)$ is generated by all Dehn twists around separating simple closed curves and all double twists around pairs of disjoint simple closed nonseparating curves that together separate (see Johnson [14]). Such a pair of non-separating curves is called a bounding pair.

Farb and Ivanov [9] introduce a graph they call the Torelli geometry. The vertices of this graph comprise all separating curves and bounding pairs in $\Sigma$, with two distinct vertices declared adjacent if their corresponding curves or bounding pairs are disjoint. Whenever $\Sigma$ has genus at least 3 this graph is connected, as follows from Masur and Schleimer [17]. For this reason, let us take $S$ to be the collection of primitive positive Dehn twists about separating curves and double twists around bounding pairs. The corresponding commutativity graph $K(\mathcal{I}(\Sigma), S)$ is precisely the Torelli geometry. As is the case with mapping class groups, adjacent vertices generate a rank 2 free abelian subgroup of $\mathcal{I}(\Sigma)$. We thus have:

Proposition 7 If $\Sigma$ is a closed and oriented surface of genus at least 3 , then the Torelli group $\mathcal{I}(\Sigma)$ of $\Sigma$ is not strongly relatively hyperbolic. 


\subsection{The special automorphism group of a free group}

In this subsection, we use the notation and basic results from Gersten [10]. Let $\mathbf{F}_{n}$ be the free group on $n$ generators and consider the automorphism group $\operatorname{Aut}\left(\mathbf{F}_{n}\right)$ of $\mathbf{F}_{n}$. Abelianisation gives a surjective homomorphism

$$
\operatorname{Aut}\left(\mathbf{F}_{n}\right) \rightarrow \operatorname{Aut}\left(\mathbb{Z}^{n}\right)=\operatorname{GL}(n, \mathbb{Z})
$$

There is a natural surjective homomorphism from $\operatorname{GL}(n, \mathbb{Z})$ onto $\mathbb{Z}_{2}$ which assigns, to any given element of $\operatorname{GL}(n, \mathbb{Z})$, the sign of its determinant. Thus, we have a surjective homomorphism

$$
\varphi: \operatorname{Aut}\left(\mathbf{F}_{n}\right) \rightarrow \mathbb{Z}_{2}
$$

The special automorphism group of $\mathbf{F}_{n}$ is $\operatorname{Aut}^{+}\left(\mathbf{F}_{n}\right)=\operatorname{ker}(\varphi)$, and has the following finite presentation in terms of right Nielsen maps: Let $X$ be a free basis for $\mathbf{F}_{n}$ and let $E=X \cup X^{-1}$. Given $a, b \in E$ with $a \neq b, b^{-1}$, define the right Nielsen map $R_{a b}$ for $a, b$ by $R_{a b}: \mathbf{F}_{n} \rightarrow \mathbf{F}_{n}$, where $R_{a b}(a)=a b$ and $R_{a b}(c)=c$ for $c \neq a, a^{-1}$.

Gersten [10] shows that $\operatorname{Aut}^{+}\left(\mathbf{F}_{n}\right)$ is generated by the finite set

$$
\left\{R_{a b} \mid a, b \in E \text { with } a \neq b, b^{-1}\right\}
$$

of right Nielsen maps, and that the following relation holds:

$$
\left[R_{a b}, R_{c d}\right]=1 \text { if } a \neq c, d, d^{-1} \text { and } b \neq c, c^{-1}
$$

Proposition 8 If $n \geq 3$, then $\operatorname{Aut}^{+}\left(\mathbf{F}_{n}\right)$ is not strongly relatively hyperbolic.

Proof Given $a, b \in E$ with $a \notin\left\{b, b^{-1}\right\}$, define also the left Nielsen map $L_{a b}: \mathbf{F}_{n} \rightarrow \mathbf{F}_{n}$ by $L_{a b}(a)=b a$ and $L_{a b}(c)=c$ for all $c \notin\left\{a, a^{-1}\right\}$. Observe that $\left[R_{a b}, L_{c d}\right]=1$ for all $a, b, c, d \in E$ with $a \notin\left\{d, d^{-1}\right\}$ and $b \notin\left\{c, c^{-1}\right\}$. Let

$$
S=\left\{R_{a b} \mid a, b \in E \text { with } a \neq b, b^{-1}\right\} \cup\left\{L_{a b} \mid a, b \in E \text { with } a \neq b, b^{-1}\right\}
$$

be the set of all of right and left Nielsen maps. The set $S$ generates $\operatorname{Aut}^{+}\left(\mathbf{F}_{n}\right)$, as immediately follows from the comments above. We first show that $K\left(\operatorname{Aut}^{+}\left(\mathbf{F}_{n}\right), S\right)$ is connected for $n \geq 3$. From the commutativity relation between right and left Nielsen maps described above, it suffices to show that any two vertices corresponding to right Nielsen maps can be connected by a path in $K\left(\operatorname{Aut}^{+}\left(\mathbf{F}_{n}\right), S\right)$.

Let $R_{a b}$ and $R_{c d}$ be two vertices of $K\left(\operatorname{Aut}^{+}\left(\mathbf{F}_{n}\right), S\right)$. We have $R_{a b}$ and $R_{c d}$ are adjacent in $K\left(\operatorname{Aut}^{+}\left(\mathbf{F}_{n}\right), S\right)$ unless $a \in\left\{c, d, d^{-1}\right\}$ or $b \in\left\{c, c^{-1}\right\}$. Let us consider the case $a=c$, noting 
the remaining cases can be similarly proven. In this case, $a \notin\{b, d\}$ and the sequence of generators $R_{a b}, L_{a b}, R_{a d}$ represents a path in $K\left(\operatorname{Aut}^{+}\left(\mathbf{F}_{n}\right), S\right)$ from $R_{a b}$ to $R_{a d}$.

Also, note that the Nielsen maps corresponding to two adjacent vertices of $K\left(\operatorname{Aut}^{+}\left(\mathbf{F}_{n}\right), S\right)$ generate a rank 2 free abelian subgroup of $\mathrm{Aut}^{+}\left(\mathbf{F}_{n}\right)$, since they commute, they both have infinite order and neither is a power of the other. The result now follows from Theorem 2.

Let $\operatorname{Out}^{+}\left(\mathbf{F}_{n}\right)=\operatorname{Aut}^{+}\left(\mathbf{F}_{n}\right) / \operatorname{Inn}\left(\mathbf{F}_{n}\right)$ be the special outer automorphism group of $\mathbf{F}_{n}$. It is immediate that the natural surjective homomorphism from $\operatorname{Aut}^{+}\left(\mathbf{F}_{n}\right)$ to $\operatorname{Out}^{+}\left(\mathbf{F}_{n}\right)$ preserves the connectivity of the commutativity graph for $\operatorname{Aut}^{+}\left(\mathbf{F}_{n}\right)$. We thus note the following:

Corollary $9 \mathrm{Out}^{+}\left(\mathbf{F}_{n}\right)$ is not strongly relatively hyperbolic for $n \geq 3$.

Restricting the surjective homomorphism $\operatorname{Aut}\left(\mathbf{F}_{n}\right) \rightarrow \operatorname{GL}(n, \mathbf{Z})$ to $\operatorname{Aut}^{+}\left(\mathbf{F}_{n}\right)$, we obtain a homomorphism $\operatorname{Aut}^{+}\left(\mathbf{F}_{n}\right) \rightarrow \mathrm{SL}(n, \mathbf{Z})$. The generating set $S=\left\{E_{a b} \mid a, b \in E\right.$ with $\left.a \neq b, b^{-1}\right\}$ projects onto a generating set $\bar{S}$ for $\operatorname{SL}(n, \mathbf{Z})$ whose elements have infinite order in $\operatorname{SL}(n, \mathbf{Z})$, as immediately follows from the definition of the Nielsen maps. Also, $K(\operatorname{SL}(n, \mathbf{Z}), \bar{S})$ is connected, since $K\left(\operatorname{Aut}^{+}\left(\mathbf{F}_{n}\right), S\right)$ is connected. We thus have:

Corollary $10 \mathrm{SL}(n, \mathbf{Z})$ is not strongly relatively hyperbolic for $n \geq 3$.

We note that the results in this section are implicit in Karlsson and Noskov [15]. See also Behrstock, Druţu and Mosher [1] for an alternative proof.

\section{References}

[1] J. A. Behrstock, C.Druţu and L. Mosher, Thick metric spaces, relative hyperbolicity, and quasi-isometric rigidity. Preprint, December 2005.

[2] B. H. Bowditch, Hyperbolic 3-manifolds and the geometry of the curve complex, in "European Congress of Mathematics, Stockholm, June 27 - July 2, 2004" (ed. A.Laptev) European Mathematical Society Publishing House (2005) 103-115.

[3] B. H. Bowditch, Relatively hyperbolic groups, preprint (1997).

[4] M. Culler and K. Vogtmann, A group-theoretic criterion for property FA, Proc. Amer. Math. Soc. 124, (1998), 677-683.

[5] M. Dadarlat and E. Guentner, Uniform embeddability of relatively hyperbolic groups, arXiv:math.GR/0501495.

[6] F. Dahmani, Classifying spaces and boundaries for relatively hyperbolic groups, Proc. London Math. Soc. 86 (2003), 666-684.

[7] B. Farb, Relatively hyperbolic groups, Geom. Funct. Anal. 8 (1998), 1-31.

[8] B. Farb, J. Franks, Groups of homeomorphisms of one-manifolds, I: Actions of nonlinear groups. Preprint, 2001.

[9] B. Farb, N. V. Ivanov, The Torelli geometry and its applications: research announcement, Math. Res. Lett. 12 (2005), 293-301.

[10] S. M. Gersten, A presentation for the special automorphism group of a free group. J. Pure Appl. Algebra 33 (1984), no. 3, 269-279. 
[11] M. Gromov, Hyperbolic Groups, in Essays in group theory, 75-263, Math. Sci. Res. Inst. Publ. 8, Springer, New York, 1987.

[12] W. J. Harvey, Boundary structure of the modular group : in Riemann surfaces and related topics: Proceedings of the 1978 Stony Brook Conference (ed. I. Kra, B. Maskit), Annals of Mathematical Studies 97, Princeton University Press (1981) 245-251.

[13] N. V. Ivanov, Mapping class groups, in Handbook of geometric topology, edited by R. Daverman and R. Sher, Elsevier, 2001, 523-633.

[14] D. L. Johnson, The structure of the Torelli group. I. A finite set of generators for I. Ann. of Math. (2) 118 (1983), no. 3, 423-442.

[15] A. Karlsson and G. Noskov, Some groups have only elementary actions on metric spaces with hyperbolic boundaries, Geom. Dedicata 104 (2004), 119-137.

[16] H. A. Masur and Y. N. Minsky, Geometry of the complex of curves I: Hyperbolicity, Invent. Math. 138 (1999), 103-149.

[17] H. A. Masur and S. Schleimer, The pants complex has only one end, in Spaces of Kleinian groups (eds. Y. Minsky, M. Sakuma, C. Series). LMS Lecture Notes 329.

[18] D. V. Osin, Asymptotic dimension of relatively hyperbolic groups. Int. Math. Res. Not. 35 (2005), $2143-2161$.

[19] D. V. Osin, Relatively hyperbolic groups: intrinsic geometry, algebraic properties, and algorithmic problems. Mem. Amer. Math. Soc. 179 (2006), no. 843, vi+100 pp.

[20] N. Ozawa, Boundary amenability of relatively hyperbolic groups. Topology Appl. 153 (2006), no. 14, $2624-2630$.

[21] A. Szczepański, Relatively hyperbolic groups, Michigan Math. J. 45 (1998), 611-618.

J. W. Anderson (corresponding author)

School of Mathematics

University of Southampton

Southampton SO17 1BJ

England

j.w.anderson@soton.ac.uk

J. Aramayona

Mathematics Institute

University of Warwick

Coventry CV4 7AL

England

jaram@maths.warwick.ac.uk

K. J. Shackleton

School of Mathematics

University of Southampton

Southampton SO17 1BJ

England

kjs2006@alumni.soton.ac.uk 